\title{
ON A THEOREM OF DUREN, SHAPIRO AND SHIELDS
}

\author{
SHINJI YAMASHITA
}

ABSTRACT. We shall show that, an extension of the theorem of Duren, Shapiro and Shields on the univalence of a function $f$ holomorphic in the unit disk $D$, still remains of significance, if the power $\alpha \in(0,1)$ in

$$
\sup _{z \in D}\left(1-|z|^{2}\right)^{\alpha}\left|f^{\prime \prime}(z) / f^{\prime}(z)\right|
$$

is small enough.

Let $f$ be a function nonconstant and holomorphic in $D=\{|z|<1\}$, let $\alpha>0$, and let

$$
\|f\|_{\alpha}=\sup _{z \in D}\left(1-|z|^{2}\right)^{\alpha}\left|f^{\prime \prime}(z) / f^{\prime}(z)\right| \text {. }
$$

It was proved by P. L. Duren, H. S. Shapiro and A. L. Shields [3, Theorem 2] that if

$$
\|f\|_{1} \leqslant 2(\sqrt{5}-2)=0.47 \ldots,
$$

then $f$ is univalent in $D$. This was improved later by $\mathbf{J}$. Becker [1, Corollary 4.1, p. 36] in the sense that if $\|f\|_{1} \leqslant 1$, then $f$ is univalent in $D$. Therefore, if $\alpha \in[0,1]$, and if $\|f\|_{\alpha} \leqslant 1$, then $f$ is univalent in $D$ because $\|f\|_{\alpha}>\|f\|_{1}$.

We shall show, nevertheless, the method found by Duren, Shapiro and Shields still remains available for small $\alpha>0$.

TheOREM. Let $f$ be a function nonconstant and holomorphic in D. Suppose that $\alpha \in[0,1]$, and suppose that

$$
\|f\|_{\alpha} \leqslant 2\left(\sqrt{4^{\alpha}+2-\alpha}-2^{\alpha}\right) \equiv k_{\alpha} .
$$

Then $f$ is univalent in $D$.

Our theorem is thus significant for $k_{\alpha}>1$; this is the case for $0<\alpha<\alpha_{0}$, where $\alpha_{0}$ is the constant satisfying $2^{\alpha_{0}}+\alpha_{0}=7 / 4$. We note that $\alpha_{0}=$ $0.416 \ldots$; the detailed calculation is due to the referee. The constant $k_{1}$ is the constant found by Duren, Shapiro and Shields.

We remark that the constant $k_{0}=2(\sqrt{3}-1)=1.46 \ldots$ is not good. In effect, it is known that if $\|f\|_{0} \leqslant 2 \sqrt{2}=2.82 \ldots$, then $f$ is univalent in $D$; see [4, Problem 2, p. 179] (this Problem 2 is solved on making use of the Schwarz and Pick lemma, together with the theorem of V. V. Pokornyi, cited in [4, Problem 1, p. 179]).

Received by the editors March 15, 1978.

AMS (MOS) subject classifications (1970). Primary 30A36. 
We begin with

LEMMA. Let $g$ be a function holomorphic in $D$, and let $\alpha>0$. Then

$$
\sup _{z \in D}\left(1-|z|^{2}\right)^{1+\alpha}\left|g^{\prime}(z)\right|<2^{1+\alpha} \sup _{z \in D}\left(1-|z|^{2}\right)^{\alpha}|g(z)| \text {. }
$$

Proof. We may assume that the supremum in the right-hand side of (1), denoted by $M$, is finite, because the case where $M=+\infty$ is obvious. For each $z \in D$, we let

Then

$$
\rho=\left[\frac{1}{2}\left(1+r^{2}\right)\right]^{1 / 2}, \quad r=|z|
$$

Since

$$
\left(1-r^{2}\right)^{1+\alpha}=2^{1+\alpha}\left(\rho^{2}-r^{2}\right)\left(1-\rho^{2}\right)^{\alpha}
$$

$$
g^{\prime}(z)=(2 \pi i)^{-1} \int_{|w|=\rho} g(w)(w-z)^{-2} d w
$$

it follows that

$$
\begin{aligned}
\left(1-|z|^{2}\right)^{1+\alpha}\left|g^{\prime}(z)\right|< & 2^{1+\alpha}\left(\rho^{2}-r^{2}\right)\left(1-\rho^{2}\right)^{\alpha}\left(\sup _{|w|=\rho}|g(w)|\right) \\
& \times\left[(2 \pi)^{-1} \int_{0}^{2 \pi}\left|\rho e^{i \theta}-z\right|^{-2} \rho d \theta\right] \\
& <2^{1+\alpha} M\left[(2 \pi)^{-1} \int_{0}^{2 \pi}\left(\rho^{2}-r^{2}\right)\left|\rho e^{i \theta}-z\right|^{-2} d \theta\right]=2^{1+\alpha} M .
\end{aligned}
$$

We thus obtain (1).

Proof of Theorem. It follows from the Lemma, applied to $g=f^{\prime \prime} / f^{\prime}$, that

$$
\left(1-|z|^{2}\right)^{1+\alpha}\left|\left(f^{\prime \prime}(z) / f^{\prime}(z)\right)^{\prime}\right| \leqslant 2^{1+\alpha}\|f\|_{\alpha} \leqslant 2^{1+\alpha} k_{\alpha}, \quad z \in D .
$$

On the other hand, it follows from $1+\alpha \geqslant 2 \alpha$, that

$$
\left(1-|z|^{2}\right)^{1+\alpha}\left|f^{\prime \prime}(z) / f^{\prime}(z)\right|^{2}<\|f\|_{\alpha}^{2}<k_{\alpha}^{2}, \quad z \in D .
$$

Therefore, we obtain

$$
\left(1-|z|^{2}\right)^{1+\alpha}\left|S_{f}(z)\right|<2^{1+\alpha} k_{\alpha}+\frac{1}{2} k_{\alpha}^{2}=2(2-\alpha), \quad z \in D,
$$

where $S_{f}=\left(f^{\prime \prime} / f^{\prime}\right)^{\prime}-\frac{1}{2}\left(f^{\prime \prime} / f^{\prime}\right)^{2}$ is the Schwarzian derivative of $f$.

Now, P. R. Beesack [2, (2.6), p. 217, and (2.8), p. 218] proved that if there exists $\lambda \in[1,2]$ such that

$$
\left(1-|z|^{2}\right)^{\lambda}\left|S_{f}(z)\right| \leqslant 2(3-\lambda), \quad z \in D
$$

then $f$ is univalent in $D$. Since $1<1+\alpha<2$, it follows from (2) that $f$ is univalent in $D$.

\section{REFERENCES}

1. Jochen Becker, Löwnersche Differentialgleichung und quasikonform fortsetzbare schlichte Funktionen, J. Reine Angew. Math. 255 (1972), 23-43. 
2. Paul R. Beesack, Nonoscillation and disconjugacy in the complex domain, Trans. Amer. Math. Soc. 81 (1950), 211-242.

3. Peter L. Duren, Harold S. Shapiro and Allen L. Shields, Singular measures and domains not of Smirnoo type, Duke Math. J. 33 (1960), 247-254.

4. Christian Pommerenke, Unioalent functions, Vandenhoeck und Ruprecht, Göttingen, 1975.

Department of Mathematics, Tokyo Metropolitan University, Furazawa, SETAGAYA-KU, TOXYY 158, JAPAN 